\title{
Dependence of Electron Density on Fermi Energy in N-Type Gallium Antimonide
}

\section{Volume 108 \\ Herbert S. Bennett and Howard Hung}

National Institute of Standards and Technology, Gaithersburg, MD 20899-8120 USA

herbert.bennett@nist.gov howard.hung@nist.gov
The majority electron density as a function of the Fermi energy is calculated in zinc blende, n-type $\mathrm{GaSb}$ for donor densities between $10^{16} \mathrm{~cm}^{-3}$ and $10^{19} \mathrm{~cm}^{-3}$. These calculations solve the charge neutrality equation self-consistently for a four-band model (three conduction sub-bands at $\Gamma, \mathrm{L}$, and $\mathrm{X}$ and one equivalent valence band at $\Gamma$ ) of GaSb. Our calculations assume parabolic densities of states and thus do not treat the density-of-states modifications due to high concentrations of dopants, many body effects, and non-parabolicity of the bands. Even with these assumptions, the results are important for interpreting optical measurements such as Raman measurements that are proposed as a nondestructive method for wafer acceptance tests.

Key words: band structure; dopants; electron density; Fermi energy; gallium antinomide; Raman measurements.

Accepted: April 11, 2003

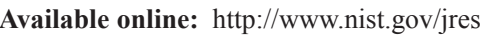

\section{Introduction}

Most interpretations of optical measurements on compound semiconductors such as GaSb require physical models and associated input parameters that describe how carrier densities vary with dopant concentrations and measured Fermi energies. In this paper, we report on a method that gives closed form analytic expressions for the carrier densities in the conduction sub-bands for GaSb at room temperature. The method is based on an iterative and self-consistent solution of the charge neutrality equation with full Fermi-Dirac statistics for the carriers at finite temperature and on the use of statistical analyses to give analytic expressions that represent the calculated data sets.

The method reported here is related to earlier work on n-type GaAs presented in reference [1]. Reference [1] gives the results predicted by an effective two-band model, one equivalent conduction band and one equivalent valence band at $\Gamma$, that includes the densities of states modifications due to high concentrations of dopants and due to many-body effects associated with carrier-carrier interactions. The method given below for $\mathrm{GaSb}$ is a four-band model. But, because of computational limitations, it does not include the densities of states modifications due to high concentrations of dopants and due to many-body effects.

\section{Theory}

The electron $n$ and hole concentrations $h$ in units of $\mathrm{cm}^{-3}$ at thermal equilibrium are given, respectively, by

$$
n=\int_{-\infty}^{+\infty} f_{0}(E) \rho_{\mathrm{c}}(E) \mathrm{d} E \text { and } h=\int_{-\infty}^{+\infty}\left[1-f_{0}(E)\right] \rho_{\mathrm{v}}(E) \mathrm{d} E
$$

where $f_{0}(E)=\left\{1+\exp \left[\left(E-E_{\mathrm{F}}\right) / k_{\mathrm{B}} T\right]\right\}^{-1}$ is the FermiDirac distribution function, $E_{\mathrm{F}}$ is the Fermi energy in $\mathrm{eV}, \rho_{\mathrm{c}}(E)$ and $\rho_{\mathrm{v}}(E)$ are, respectively, the electron den- 
sity of states for the conduction band and the hole density of states for the valence band, $k_{\mathrm{B}}$ is the Boltzmann constant, and $T$ is the temperature in kelvins. The calculations incorporate the Thomas-Fermi expression for the screening radius,

$$
r_{\mathrm{s}}^{2}=-\frac{4 \pi \mathrm{e}^{2}}{\varepsilon \varepsilon_{0}} \int_{-\infty}^{+\infty} \frac{\mathrm{d} f_{0}(E)}{\mathrm{d} E}\left[\rho_{\mathrm{c}}(E)-\rho_{\mathrm{v}}(E)\right] \mathrm{d} E
$$

and the charge neutrality condition

$$
N_{\mathrm{I}}=n-h,
$$

to compute self-consistently the Fermi energy $E_{\mathrm{F}}$ and the screening radius $r_{\mathrm{s}}$ for given values of the ionized dopant concentration $N_{\mathrm{I}}$ and temperature $T$. The static dielectric constant is $\varepsilon$ and the permittivity of free space is $\varepsilon_{0}$. The ionized dopant concentration is positive for $\mathrm{n}$ type material (donor ions) and negative for p-type material (acceptor ions). The results reported here are for uncompensated n-type material. The results for the screening radius $r_{\mathrm{s}}$ are not reported here because they are not needed to extract carrier concentrations from Raman measurements.

In this paper, we use the four-band model that has three conduction sub-bands centered at the $\Gamma, \mathrm{L}$, and $\mathrm{X}$ symmetry points in the Brillouin zone and one equivalent valence band centered at the $\Gamma$ symmetry point. We do not include the detailed nonparabolicity of the $\mathrm{GaSb}$ energy bands at $\Gamma$. Unlike GaAs, the GaSb conduction $\Gamma, \mathrm{L}$, and $\mathrm{X}$ sub-band masses and energy spacings are such that for donor densities of technological interest, the conduction sub-band at $\mathrm{L}$ is the one that is most populated. The non-parabolicity of the conduction $\Gamma$ sub-band in GaAs is discussed in Ref. [2]. If we were to use the Kane three level $k \cdot p$ model [2], which does not include the conduction sub-bands at $\mathrm{L}$ and $\mathrm{X}$, we would be able to include the non-parabolicity of the conduction $\Gamma$ sub-band. However, because the conduction $\Gamma$ sub-band band in GaSb is not the dominant band for determining the Fermi energy, its non-parabolicity correction may not have a significant effect on the results given below and may lie within the uncertainties associated with the band masses quoted in the literature for GaSb.

The heavy hole mass $m_{\mathrm{hh}}$ and light hole mass $m_{\mathrm{lh}}$ for the two degenerate sub-bands at the top of the valence band are combined to give an effective mass

$$
m_{\mathrm{v} \Gamma}=\left(m_{\mathrm{hh}}^{3 / 2}+m_{\mathrm{lh}}^{3 / 2}\right)^{2 / 3},
$$

for the valence topmost sub-band. The values of these parameters are given in Table 1.

The zero of energy is at the bottom of the conduction $\Gamma$ sub-band. The bottoms of the conduction $\mathrm{L}$ and $\mathrm{X}$ sub-bands are, respectively, at $E_{\mathrm{cL}}$ and $E_{\mathrm{cX}}$. The top of the degenerate valence $\Gamma$ sub-band is at $-E_{\mathrm{G}}$, where $E_{\mathrm{G}}$ is the bandgap of GaSb. The split-off valence sub-band at $\Gamma$ due to spin-orbit coupling and the non-parabolicity factor of the conduction $\Gamma$ sub-band are neglected. The probabilities for typical carriers in equilibrium to occupy appreciably these states are low. This means that the Fermi energies should be sufficiently above the valence sub-band maximum at $\Gamma$. Placing exact limits on the Fermi energies for which the four-band model is valid would be tenuous, because knowledge of how the various sub-bands move relative to one another due to the dopant concentrations considered here and due to many body effects is not adequate.

The general expression [3] for the temperature dependence of conduction sub-band minima relative to the top of the valence band at $\Gamma$ is

$$
E_{i}=E_{i 0}-\left[A_{i} T^{2} /\left(T+B_{i}\right)\right]
$$

in units of $\mathrm{eV}$, where $i=\Gamma$, $\mathrm{L}$, or $\mathrm{X}$. The values for the coefficients $E_{i 0}, A_{i}$, and $B_{i}$ are listed in Table 2.

The general expression for the parabolic densities of states for electrons and holes per band extrema and per spin direction is given by

$$
\rho(E)=\frac{N_{\mathrm{e}} 4 \pi V \sqrt{E}}{\left(8 \pi^{3}\right)\left(\hbar^{2} / 2 m^{*} m_{0}\right)^{3 / 2}},
$$

where $N_{\mathrm{e}}$ is the number of equivalent ellipsoids in the first Brillouin zone, the volume of the unit cell is $V=a_{\mathrm{L}}{ }^{3}, a_{\mathrm{L}}$ is the lattice constant, $m^{*}$ is one of the effective masses listed in Table 1 for the appropriate band extrema, and $m_{0}$ is the free electron mass. Because eight permutations of the wave vector in the (111) direction exist, there are eight $\mathrm{L}$ sub-band ellipsoids with centers located near the boundary of the first Brillouin zone. Also, because six permutations of the wave vector in the (100) direction exist, there are six X sub-band ellipsoids with centers located near the boundary of the first Brillouin zone. Since about half of each ellipsoid is in the neighboring zone, the number of equivalent subbands $N_{\mathrm{cL}}$ for the L sub-band is four and the number of equivalent sub-bands $N_{\mathrm{cX}}$ for the $\mathrm{X}$ sub-band is three.

In terms of a four-band model for room temperature n-type GaSb, the total density of states $\rho_{\mathrm{c}}(E)$ for the majority carrier electrons in n-type GaSb then becomes 
Table 1. Input parameters for intrinsic zinc blende GaSb at $300 \mathrm{~K}$. The energies of the extrema of the conduction and valence sub-bands are referenced to the bottom of the conduction sub-band at the $\Gamma$ symmetry point in the Brillouin zone of the reciprocal lattice space. The mass of the free electron is $m_{0}$. These data are from Ref. [3]

\begin{tabular}{lll}
\hline \hline Parameter & Symbol & Value \\
\hline Lattice constant & $a_{\mathrm{L}}$ & $6.09593 \times 10^{-8}$ \\
Dielectric constant in vacuum & $\varepsilon$ & $8.854 \times 10^{-12}$ \\
Static dielectric constant & $\varepsilon_{0}$ & 15.7 \\
Bandgap & $E_{\mathrm{G}}=\left|E_{\mathrm{v} \Gamma}\right|$ & 0.726 \\
Bottom of the conduction L sub-band & $E_{\mathrm{cL}}$ & 0.084 \\
Bottom of the conduction X sub-band & $E_{\mathrm{vX}}$ & 0.31 \\
Top of the degenerate valence $\Gamma$ sub-band & $-E_{\mathrm{v} \Gamma}$ & -0.726 \\
Spin-orbit splitting & $E_{\mathrm{so}}$ & 0.80 \\
Top of the split-off (spin-orbit splitting) valence $\Gamma$ sub-band & $-E_{\mathrm{so} \Gamma}=-E_{\mathrm{v} \Gamma}-E_{\mathrm{so}}$ & -1.526 \\
Effective mass of conduction $\Gamma$ sub-band & $m_{\mathrm{c} \Gamma}$ & 0.041 \\
Transverse $L$ sub-band mass & $m_{\mathrm{tL}}$ & 0.11 \\
Longitudinal $L$ sub-band mass & $m_{\mathrm{lL}}$ & 0.95 \\
Effective mass of conduction $L$ sub-band & $\left.m_{\mathrm{cL}}=\left(m_{\mathrm{lL}} m_{\mathrm{tL}}\right)^{2}\right)^{1 / 3}$ & 0.226 \\
Transverse X sub-band mass & $m_{\mathrm{tX}}$ & 0.22 \\
Longitudinal X sub-band mass & $m_{\mathrm{lX}}$ & 1.51 \\
Effective mass of conduction X sub-band & $m_{\mathrm{cX}}=\left(m_{\mathrm{lX}} m_{\mathrm{tX}}{ }^{2}\right)^{1 / 3}$ & 0.418 \\
Light hole mass of degenerate valence $\Gamma$ sub-band & $m_{\mathrm{lh}}$ & 0.05 \\
Heavy hole mass of degenerate valence $\Gamma$ sub-band & $m_{\mathrm{hh}}$ & 0.4 \\
Effective mass of degenerate valence $\Gamma$ sub-band & $m_{\mathrm{v} \Gamma}$ & 0.41 \\
Splitoff band mass of the valence sub-band at $\Gamma$ & $m_{\mathrm{so}}$ & 0.14 \\
Number of equivalent conduction $L$ sub-bands & $N_{\mathrm{cL}}$ & 4 \\
Number of equivalent conduction X sub-bands & $N_{\mathrm{cX}}$ & 3 \\
\hline
\end{tabular}

$$
\rho_{\mathrm{c}}(E)=\rho_{\mathrm{c \Gamma}}(E)+\rho_{\mathrm{cL}}(E)+\rho_{\mathrm{cX}}(E)
$$

where $\rho_{\mathrm{cr}}(E), \rho_{\mathrm{cL}}(E)$, and $\rho_{\mathrm{cx}}(E)$ are the sub-band densities of states for the conduction $\Gamma, \mathrm{L}$, and $\mathrm{X}$ sub-bands with effective masses of $m_{\mathrm{c} \Gamma}, m_{\mathrm{cL}}$, and $m_{\mathrm{cX}}$, respectively. The density of states for the minority carrier holes is

$$
\rho_{\mathrm{v}}(E)=\rho_{\mathrm{v} \Gamma}(E)
$$

with an effective mass of $m_{\mathrm{v} \Gamma}$.

\section{Results}

Tables 1 and 2 contain the input parameters for the calculations of the Fermi energy as a function of the dopant donor density. We solve self-consistently, by means of an iterative procedure, Eq. (3) with Eqs. (6), (7) and (8). The independent variable is the temperature $T$. The Fermi energy is varied for a given temperature until Eq. (3) is satisfied. Figure 1 presents the calculated data graphically for 28 values of donor densities between $10^{16} \mathrm{~cm}^{-3}$ and $10^{19} \mathrm{~cm}^{-3}$. Figure 2 gives the electron densities in the conduction sub-bands at $\Gamma$ and $\mathrm{L}$ and the total electron density as functions of the Fermi energy. Figure 2 does not show the electron density in the conduction sub-band at $\mathrm{X}$, because it is less
Table 2. Coefficients for the temperature dependence of the conduction band extrema that are used in Eq. (5). These data are from Ref. [3]

\begin{tabular}{llll}
\hline \hline Parameter & Symbol & Value & Units \\
\hline$\Gamma$ sub-band & $E_{\Gamma 0}$ & 0.813 & $\mathrm{eV}$ \\
$\Gamma$ sub-band & $A_{\Gamma}$ & $3.78 \times 10^{-4}$ & $\mathrm{eV} / \mathrm{K}$ \\
$\Gamma$ sub-band & $B_{\Gamma}$ & 94. & $\mathrm{~K}$ \\
L sub-band & $E_{\mathrm{L} 0}$ & 0.902 & $\mathrm{eV}$ \\
L sub-band & $A_{\mathrm{L}}$ & $3.97 \times 10^{-4}$ & $\mathrm{eV} / \mathrm{K}$ \\
L sub-band & $B_{\mathrm{L}}$ & 94. & $\mathrm{~K}$ \\
X sub-band & $E_{\mathrm{X} 0}$ & 1.142 & $\mathrm{eV}$ \\
$X$ sub-band & $A_{\mathrm{X}}$ & $4.75 \times 10^{-4}$ & $\mathrm{eV} / \mathrm{K}$ \\
X sub-band & $B_{\mathrm{X}}$ & 94. & $\mathrm{~K}$ \\
\hline
\end{tabular}

than $10^{-3}$ times the total electron density. Because $m_{\mathrm{c} \Gamma}<<m_{\mathrm{cL}}$ and $E_{\mathrm{cL}}$ is much closer to $E_{\mathrm{c} \Gamma}$ than it is to $E_{\mathrm{cX}}$, the electron density in the conduction $\mathrm{L}$ sub-band exceeds the electron density in the conduction $\Gamma$ subband at room temperature. The solid curve in Fig. 2 is the same curve as given in Fig. 1. Figure 2 shows that the majority of electrons is in the conduction L subband and that the density of electrons in the L sub-band approaches the total density of electrons as the donor density approaches $10^{19} \mathrm{~cm}^{-3}$. Hence, even though $\mathrm{GaSb}$ is intrinsically a direct semiconductor, the results from Fig. 2 suggest that electrons for n-type GaSb in 


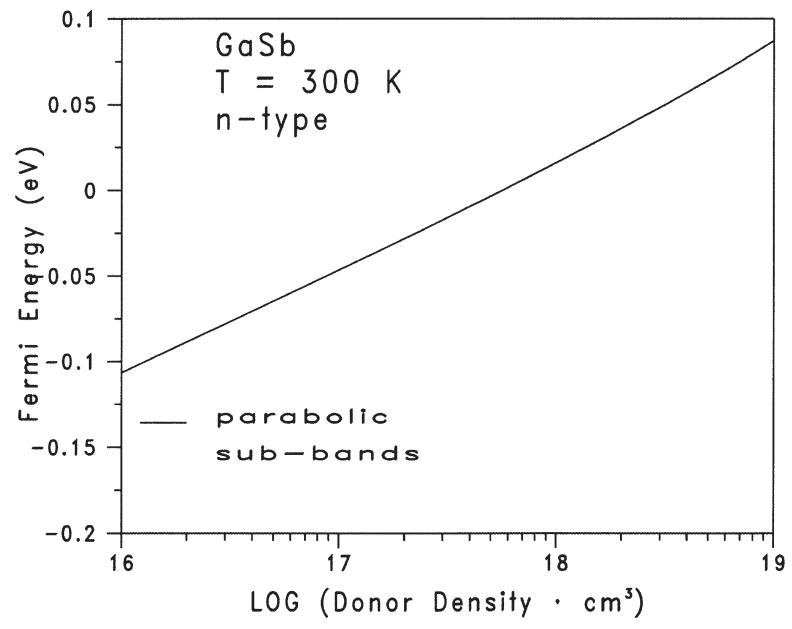

Fig. 1. The calculated Fermi energy for n-type GaSb at $300 \mathrm{~K}$ as a function of the donor density. The Fermi energy is relative to the majority conduction band edge at the $\Gamma$ symmetry point in the first Brillouin zone.

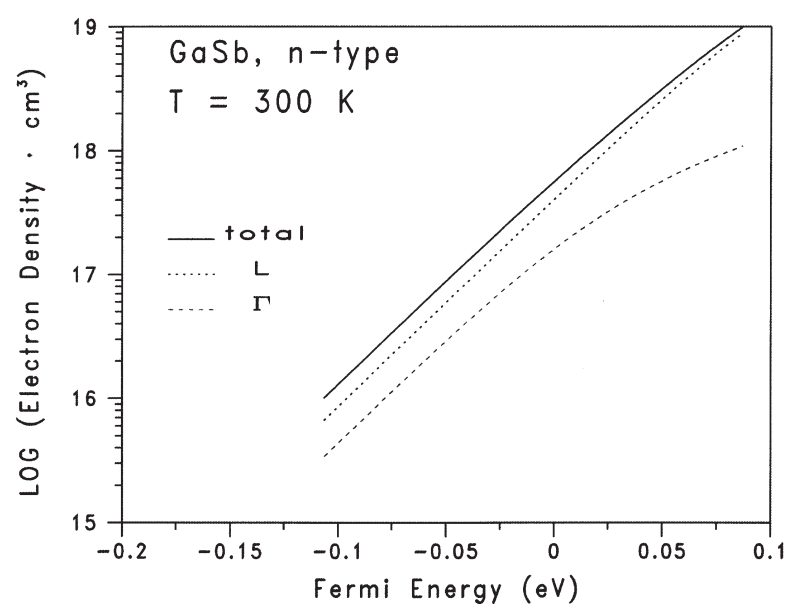

Fig. 2. The calculated electron densities in the conduction sub-bands at $\Gamma$ and $L$ and the total electron density as functions of the Fermi energy. The Fermi energy is relative to the majority conduction band edge at the $\Gamma$ symmetry point in the first Brillouin zone. the vicinity of the Fermi surface will behave as though they have many characteristics of electrons in an indirect semiconductor.

For illustrative purposes, we give here only the results for fitting the logarithm to the base 10 of the total electron density $n$ in units of $\mathrm{cm}^{-3}$ to a polynomial in $E_{\mathrm{F}}$, namely,

$$
\log _{10}\left(n \mathrm{~cm}^{-3}\right)=a_{0}+a_{1} E_{\mathrm{F}}+a_{2} E_{\mathrm{F}}^{2}+\ldots+a_{1} E_{\mathrm{F}}^{1} \ldots
$$

The analytic fits for the electron densities in the $\Gamma, \mathrm{L}$, and $\mathrm{X}$ sub-bands are available by sending an email to herbert.bennett@nist.gov. During the fitting analyses, we rely substantially on graphics and keep the number of fitting parameters to a minimum, subject to the constraint that the residual standard deviation $S_{\text {res }}$ is acceptably small, i.e., $S_{\text {res }} \leq 0.01$. The standard deviation is a measure of the "average" error in a fitted model and thereby is a metric for assessing the quality of the fit. A smaller $S_{\text {res }}$ indicates a better fit. The residual standard deviation for a model $Y^{f}=f(Z)$ is

$$
S_{\text {res }}=\sqrt{\left[\sum_{j=1}^{N}\left(Y_{j}-\bar{Y}_{j}^{f}\right)^{2} /(N-P)\right]},
$$

where $Y_{j}$ are the calculated data values, the $\bar{Y}_{j}^{f}$ are the predicted values from the fitted model, $N$ is the total number of data points (here $N=28$ ), and $P$ is the total number of parameters to be fitted in the model. We use the NIST-developed DATAPLOT [4] software for both the exploratory graphics and for the statistical analyses.

Table 3 gives the four fitting parameters for the cubic $l=3$ polynomial fit to the $\log _{10}(n)$ as shown in Eq. (9) and the associated residual standard deviation $S_{\text {res }}=$ 0.0066 . In general, the values of $S_{\text {res }}$ decrease monotonically with increasing number $l$ of terms in the polynomial given in Eq. (9). But, care must be taken to avoid fitting noise in data sets. The general guideline for

Table 3. The four fitting parameters for a cubic polynomial fit of the theoretical calculations for the total electron density in n-type, zinc blende GaSb at $300 \mathrm{~K}$ as a function of the Fermi energy relative to the bottom of the conduction $\Gamma$ sub-band. The ratio is the estimated value divided by the estimated standard deviation. The residual standard deviation is $S_{\text {res }}=0.0066$

\begin{tabular}{ccccc}
\hline \hline Fitting parameter & Estimated value & $\begin{array}{c}\text { Estimated standard } \\
\text { deviation }\end{array}$ & Units & Ratio \\
\hline$a_{0}$ & 17.7504 & $0.1774 \times 10^{-3}$ & & $1.001 \times 10^{5}$ \\
$a^{1}$ & 15.6775 & $0.5416 \times 10^{-2}$ & $\mathrm{eV}^{-1}$ & $2.895 \times 10^{3}$ \\
$a^{2}$ & -11.4745 & $0.4723 \times 10^{-1}$ & $\mathrm{eV}^{-2}$ & $-2.43 \times 10^{2}$ \\
$a^{3}$ & -41.3848 & 0.8535 & $\mathrm{eV}^{-3}$ & $-4.849 \times 10^{1}$ \\
\hline
\end{tabular}


many data sets is that when the absolute value of the ratio $R$ of the estimated parameter value divided by its estimated standard deviation is less than about 2 , then the rate of decrease in $S_{\text {res }}$ with increasing $l$ tends to decrease. For the data given in Fig. 1 , when $l=2$ or $\mathrm{P}=3, S_{\mathrm{res}}=0.0644$; when $l=3$ or $P=4, S_{\mathrm{res}}=0.0066$; and when $l=4$ or $P=5, S_{\text {res }}=0.0063$. Because the change in values of $S_{\text {res }}$ between $l=3$ and $l=4$ is not significant, we use the fitting parameters for the cubic $l=3$ case in this paper. Also, the ratio $R$ for the parameter $a_{4}$ when $l=4$ is -1.917 , and such a value for $R$ means that proceeding with higher $l$ values probably is not warranted. A figure that compares the calculated total electron density as a function of the Fermi energy with the fitting results from Eq. (9) for a cubic polynomial is not given because the two curves lie on top of one another within the line widths of each curve. Fits to the calculated electron densities for each of the conduction sub-bands are available from the author upon request. Also, since the screening radii for the carriers from Eq. (2) are not needed when interpreting the proposed measurements considered here, the corresponding screening radii are not presented in this paper.

\section{Conclusions}

The results from Sec. 3 are consistent with the findings of experimental work reported in the literature such as Refs. [5] and [6]. Interpreting experiments for $\mathrm{GaSb}$ requires at least a three-band model and under some conditions may require a four-band model. And finally, even though $\mathrm{GaSb}$ is intrinsically a direct semiconductor, our results show that electrons for n-type $\mathrm{GaSb}$ in the vicinity of the Fermi surface will have some characteristics that are similar to those for electrons in an indirect semiconductor.

\section{Acknowledgments}

The authors thank Jeremiah Lowney, formerly of NIST, for many helpful discussions and assistance during the course of this work. We acknowledge many discussions with James Maslar and Wilbur Hurst concerning physical models for interpreting Raman measurements. We thank Alan Heckert and James Filliben for help with the statistical analyses of data sets and for guidance in using DATAPLOT. We benefited substantially from having access to the NIST Centralized Computing Facility and from the support given by many NIST Information Technology Laboratory (ITL) members such as Mary-Lou Blessing, William George, Denis Lehane, William Mitchell, and James Sims.

\section{References}

[1] H. S. Bennett, J. Appl. Phys. 83, 3102 (1998).

[2] J. S. Blakemore, J. Appl. Phys. 53, R123 (1982).

[3] A. Ya. Vul, in Handbook Series on Semiconductor Parameters, Volume I, M. Levinshtein, S. Rumyantsev, and M. Shur, eds., World Scientific Publishing, Singapore (1996) pp. 125-146.

[4] J. J. Filleben and A. N. Heckert, the DATAPLOT software for graphics and detailed statistical analyses runs on both UNIX and WINTEL platforms. It has both command-line versions and graphical user interface (GUI) versions. It is available by downloading from http://www.itl.nist.gov/div898/software/dataplot/. In addition, the NIST-SEMATECH Engineering Statistics Handbook at http://www.itl.nist.gov/div898/handbook/ is based in part on DATAPLOT. This latter WWW site has tutorials that explain in some detail the statistical analysis methods used in this paper.

[5] A. Baraldi, F. Colonna, C. Ghezzi, R. Magnanini, A. Parisini, L Tarricone, A. Bosacchi, and S. Franchi, Semicond. Sci. Technol. 11, 1656 (1996).

[6] V. W. L. Chin, Solid-State Electronics 38, 59 (1995).

About the authors: Herbert S. Bennett is a NIST Fellow in the Semiconductor Electronics Division of the NIST Electronics and Electrical Engineering Laboratory. His interests include industrial consensus building for technology roadmaps on semiconductors and on optoelectronics packaging, device physics for improved understanding of electronic, optoelectronic, and magnetic materials, and assessing the quality of digital video images.

Howard Hung is a computational scientist in the Scientific Applications and Visualization Group, Mathematical and Computational Sciences Division, of the NIST Information Technology Laboratory. The National Institute of Standards and Technology is an agency of the Technology Administration, U.S. Department of Commerce. 